\title{
Upgrade of the First Level Muon Trigger in the End-Cap New Small Wheel Region of the ATLAS Detector
}

\author{
Yonathan Munwes, Tel Aviv University, Israel on behalf of the ATLAS Collaboration.
}

\begin{abstract}
The luminosity levels foreseen at the LHC after the 2018 LHC upgrade will tighten the demands on the ATLAS first level muon trigger system. A finer muon selection will be required to cope with the increased background and to keep the trigger rate for $20 \mathrm{GeV} / \mathrm{c} p_{\mathrm{T}}$ muons as before. The introduction of new detectors in the small wheel region of the end-cap muon spectrometer will allow to refine the current trigger selection, allowing to increase the rejection power for tracks not coming from the interaction point, thus to find candidate muon tracks within $1 \mathrm{mrad}$ angular resolution and within the $500 \mathrm{~ns}$ available latency. The on-detector trigger logic will require a coincidence of eight layers of small thin gap chambers detector pads to determine the trigger regions-of-interest. The charge information from the detector strips of the selected regions-of-interest will be sent to the off-detector trigger logic, which will calculate the strip centroids and extrapolate the muon tracks. The muon tracks information will be finally sent to the end-cap sector logic, which will combine the big wheel and the new small wheel trigger data, and provide the trigger muon candidates to the ATLAS central trigger.
\end{abstract}

\section{INTRODUCTION}

$\mathbf{T}$ HE ATLAS experiment [1] was designed for a broad physics programme, including precision measurements of the Standard Model (SM) and search for the Higgs boson, SUSY or Exotics new particles accessible at the Large Hadron Collider (LHC). Once such particles are discovered, their properties will have to be studied in many cases. These studies require very large data samples. For that reason, upgrades are needed of both the LHC (to increase the luminosity) and of the ATLAS experiment (to be able to cope with higher collision rates and radiation background). The large integrated luminosity eventually available will also allow access to rare processes and to higher centre-of-mass energies of colliding partons, extending the reach of the searches for heavy new particles.

The LHC complex will be upgraded in several phases. Following the long shutdown of 2013/14 (LS1), the accelerator energy will be increased to close to $7 \mathrm{TeV}$ per beam, and the luminosity will reach or exceed the nominal value of $1 \cdot 10^{34} \mathrm{~cm}^{-2} \mathrm{~s}^{-1}$. Major upgrades are planned starting from the 2018 shutdown with which the LHC luminosity will substantially exceed the initial design values. At the second long shutdown in 2018 (LS2), the accelerator luminosity will be increased to 2 to $3 \cdot 10^{34} \mathrm{~cm}^{-2} \mathrm{~s}^{-1}$, allowing the ATLAS experiment to collect $\sim 100 \mathrm{fb}^{-1}$ /year. The last upgrade will introduce significant changes to the interaction point (IP) region so that the luminosity will reach up to $7 \cdot 10^{34} \mathrm{~cm}^{-2} \mathrm{~s}^{-1}$

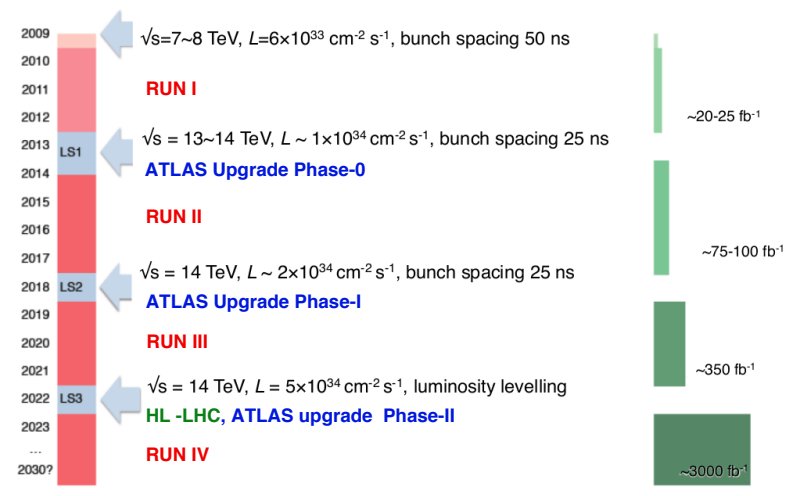

Fig. 1. An approximate timeline of the scheduled LHC and ATLAS upgrades.

(5. $10^{34} \mathrm{~cm}^{-2} \mathrm{~s}^{-1}$ with luminosity leveling) aiming to an integrated luminosity of $3000 \mathrm{fb}^{-1}$ in approximately 10 years of operation after that upgrade. Figure 1 shows an approximate timeline for the planned LHC and ATLAS upgrades. The ATLAS detector will also be upgraded following the same time schedule as the LHC upgrades.

A major goal of the Phase-I upgrade [2](2018) is improving the Level-1 trigger in order to maintain the low transverse momentum $\left(p_{T}\right)$ threshold for single leptons $(e$ and $\mu$ ) while keeping the Level-1 rate at a manageable level. Upgrades are planned for both the muon and the calorimeter trigger systems, without which the single lepton triggers would have to be either pre-scaled or its $p_{T}$ threshold raised, resulting in a significant loss of acceptance for many physics processes of interest.

\section{UPGRADE OF THE MUON SPECTROMETER: NeW SMALL WHEELS}

The Phase-I upgrade of the ATLAS muon spectrometer [3] focuses on the end-cap region. Figure 2 shows a cut-out of the ATLAS detector in z-y plane. The barrel and end-cap system consist of three stations each, measuring the muon momentum based on the curvature in the ATLAS toroid magnets.

At high luminosity the following two points are of particular importance:

- The performance of the muon tracking chambers (in particular in the end-cap region) degrades with the expected increase of cavern background rate [2]. An extrapolation from the observed rates at the lower luminosity conditions of the 2012 to high luminosity conditions indicates a substantial degradation of tracking performance, both in 


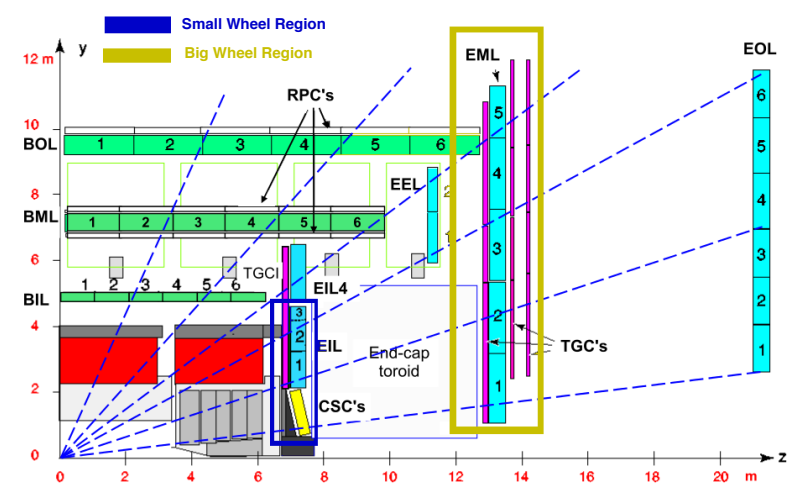

Fig. 2. A z-y view of $1 / 4$ of the ATLAS detector. The blue boxes indicate the end-cap Monitored Drift Tubes (MDT) chambers and the yellow box Cathode Strip Chambers (CSC). The green boxes are barrel MDT chambers. The trigger chambers, Resistive Plate Chambers (RPC) and Thin Gap Chamber (TGC), are indicated by the grey and the magenta boxes. This is a cut-out on the muon spectrometer at the large sectors, hence the names 'End-cap Inner Large', 'End-cap Middle Large' (EML) and 'End-cap Outer Large'.

terms of efficiency and resolution in the the inner end-cap station (at $z=7 \mathrm{~m}$ ), the 'Small Wheels'. Given that high resolution muon momentum measurement is based on the presence of measuring points at the Small Wheel level (i.e. in front of the end-cap toroid magnet), this degradation is detrimental for the performance of the ATLAS detector.

- The Level-1 muon trigger in the end-cap region is based on the track segments in the TGC chambers of the middle muon station (End-cap Muon detector, EM) located after the end-cap toroidal magnet [1]. (The TGC of the Small Wheel is only used as a rough confirmation that a particle has traversed the end-cap toroid zone. It cannot provide a precise muon trigger as, being a single plane, it cannot reconstruct a track segment.) The transverse momentum $p_{T}$ of the muon is determined by the angle of the segment with respect to the direction pointing to the interaction point. A significant part of the muon trigger rate in the end-caps are fake muons. Low energy particles generated far from the IP and out of time of the triggered bunch crossing, produce fake triggers by hitting the end-cap trigger chambers at an angle similar to that of real high $p_{T}$ muons. An analysis of 2012 data demonstrates that $90 \%$ of the muon triggers in the end-caps are fake. As a consequence the rate of Level-1 muon trigger in end-cap is 8 to 9 times higher than that in the barrel region.

Both of these two issues represent serious limitation on the ATLAS performance at high luminosity: reduced acceptance of good muon tracking, and loss of low $p_{T}$ single muon Level-1 triggers.

In order to address the two problems, ATLAS proposes to replace the present muon Small Wheels with the 'New Small Wheels' (NSW). The NSW is a set of precision tracking and trigger detectors able to work at high rates with excellent realtime space and time resolution. These detectors can provide the muon Level-1 trigger system with online track segments of good angular resolution to confirm that muon tracks originate from the IP. In this way the end-cap fake triggers will be
TABLE I

EXPECTED LEVEL- 1 RATE AT $3 \times 10^{34} \mathrm{CM}^{-2} \mathrm{~S}^{-1}$ FOR DIFFERENT $p_{\mathrm{T}}$ THRESHOLD WITH AND WITHOUT NSW. COLUMNS 3-4 SHOW THE EFFICIENCY FOR $W H$ ASSOCIATE PRODUCTION $p p \rightarrow W H$ WITH TWO DECAY MODES OF THE SM HIGGS BOSON OF THE MASS 125 GEVINTO $H \rightarrow b \bar{b}$ AND $H \rightarrow W^{+} W^{-} \rightarrow \mu \nu q q^{\prime}$.

\begin{tabular}{|l||c|c|c|}
\hline $\begin{array}{c}\text { L1MU threshold } \\
(\mathrm{GeV})\end{array}$ & $\begin{array}{c}\text { Level-1 rate } \\
(\mathrm{kHz})\end{array}$ & $\begin{array}{c}H \rightarrow b \bar{b} \\
(\%)\end{array}$ & $\begin{array}{c}H \rightarrow W^{+} W^{-} \\
(\%)\end{array}$ \\
\hline$p_{T}>20$ & 60 & 82 & 89 \\
$p_{T}>40$ & 29 & 50 & 64 \\
$p_{T}>20$ with NSW & 22 & 78 & 86 \\
\hline
\end{tabular}

considerably reduced.

\section{IMPACT ON PHYSICS PERFORMANCE}

The importance of having a high efficiency Level-1 trigger for inclusive leptons with $p_{T}<20 \sim 25 \mathrm{GeV}$ has been shown in the Higgs boson searches through the $\mathrm{WW}^{*} \rightarrow \ell \nu \ell \nu$ decay in the low Higgs mass range. This situation will become even more crucial for the high luminosity running of the LHC. Given the recent discovery of a new low mass boson, this is particularly true. Large integrated luminosity (a few $100 \mathrm{fb}^{-1}$ ) will allow the precise determination of its couplings to gauge bosons and fermions using production processes independent of the decay mode, such as Higgsstrahlung from $W(p p \rightarrow W H)$, where triggering on the $W$ by leptons of $p_{T} \sim 20 \mathrm{GeV}$ is needed to investigate the Higgs decays into $W W$, b $\bar{b}$ and $\tau^{+} \tau^{-}$final states. Table I shows a comparison of efficiency and Level-1 rate from a simulation study for the present detector and the upgrade with NSW. At the instantaneous luminosity $3 \times 10^{34} \mathrm{~cm}^{-2} \mathrm{~s}^{-1}$, the single muon Level-1 rate of the present system will become as high as $60 \mathrm{kHz}$, dominating the allowed total Level-1 rate of $100 \mathrm{kHz}$. The Level-1 rate may be reduced significantly by raising the $p_{T}$ threshold to $40 \mathrm{GeV}$, but with the high cost of reduced efficiency to the $\mathrm{WH}$ production channels. The NSW will allow ATLAS to keep the Level-1 rate, for single muons above $20 \mathrm{GeV}$, at $22 \mathrm{kHz}$ with very limited loss of efficiency. The same argument applies also to the $Z H$ associated production.

Low $p_{T}$ lepton triggers are also important for many SUSY searches where leptons are produced through cascade decays. The kinematic distribution of the final state objects depends on the mass relation between particles in the cascade as well as the couplings. Any increases in the lepton trigger threshold (and other objects like jets and $E_{T}^{\text {miss }}$ ) lead to a reduction of experimental sensitivity in the parameter space of SUSY models [2]. Efficiency is generally lower with higher $p_{T}$ threshold, and it is particularly true for the parameter space of small mass difference. Similar situations are found in other SUSY processes such as the searches for sleptons and electroweak gauginos.

\section{Muon SMall Wheel Detector Requirements For RUN III}

The highest counting rates in the muon spectrometer is observed in the Small Wheel region. A large fraction of that is due to low energy photons and neutrons. They are generated by synchronous proton collisions with the bunch crossing 
that triggers the ATLAS data-taking mechanism (in-time background), or by collisions that happen one to several bunch crossings earlier (out-of-time background). This background radiation originates from the whole ATLAS detector volume, and hence escapes capture by the radiation shielding around the beam pipe and the ATLAS calorimeters. The resulting particles are generically referred to as cavern background.

\section{A. Operating conditions for Run III}

In order to predict reliably the expected cavern background rates after the various accelerator upgrades, two elements are required: an accurate measurement of the observed background at the current operational conditions and a reliable MonteCarlo extrapolation to higher energy and luminosity. Figure 3 shows the ratio of the measured hit rates to the corresponding simulation values for the existing muon Monitored Drift Tubes (MDT) chambers. This ratio is in general between 0.5 and 1.4. As a consequence this current background simulation can be reliably used to predict counting rates in the muon system for different detector geometries and beam energies.

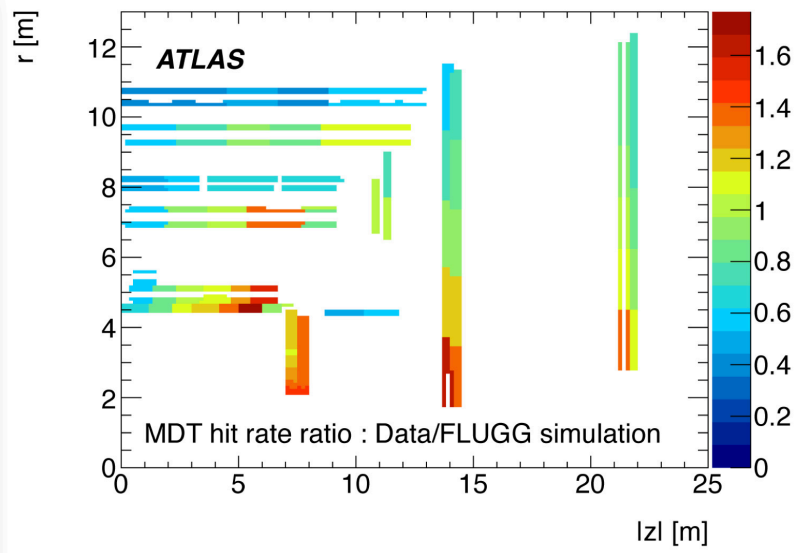

Fig. 3. Ratio of measured to simulated MDT hit rate during a $7 \mathrm{TeV}$ run at an average luminosity of $1.9 \times 10^{33} \mathrm{~cm}^{-2} \mathrm{~s}^{-1}$ (50 ns bunch spacing) [4].

The background level is a steep function of the distance from the beam line, being highest for the detectors closer to the beam pipe. Direct hit rate measurements using the muon detector have been performed at the current luminosities to study this dependency. Figure 4 (a) shows the measurements in the Small Wheel region for MDT and CSCs (Cathode Strip Chambers), used, instead of MDT, in the inner part of the present end-cap Small Wheel (since they can cope better with high radiation environments). The discontinuity at $\mathrm{R}=210 \mathrm{~cm}$ is caused by the different sensitivity of MDT and CSC to cavern background particles. The expectation by FLUGG [5] simulation is also shown in the same figure. The measured radial dependence reasonably agrees with MonteCarlo predictions. The measured results are then used to estimate the background rate on the Small Wheel as a function of radial distance from the beams, assuming that the same technology is used over the full acceptance of the Small Wheel. In Fig. 4 (b) the observed rates in MDT $(\mathrm{R}>210 \mathrm{~cm})$ and CSC $(\mathrm{R}<200 \mathrm{~cm})$ are scaled to the value corresponding to the nominal luminosity of $1 \times 10^{34} \mathrm{~cm}^{-2} \mathrm{~s}^{-1}$. In addition the CSC curve is scaled to have the same value as the MDTs one at their boundary to simulate the condition of an all MDT-type detector scenario. Similarly, the MDT curve is scaled to simulate the all CSC-type detector case. The difference between these two curves indicates a possible dependency of the background hit rate on the detector technology. Figure 4 (b) indicates that at a luminosity of $1 \times 10^{34} \mathrm{~cm}^{-2} \mathrm{~s}^{-1}$ the current MDT system will operate near its limits, and well beyond that for a luminosity of $7 \times 10^{34} \mathrm{~cm}^{-2} \mathrm{~s}^{-1}$.

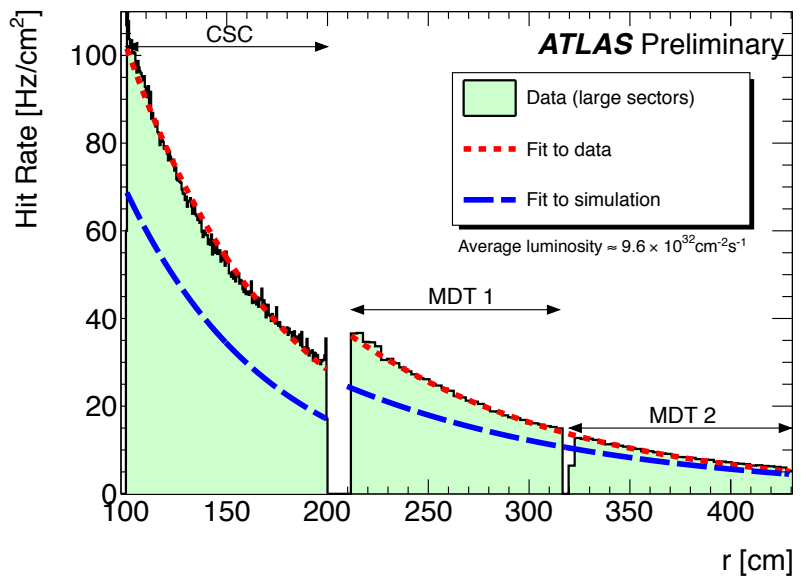

(a) $\mathcal{L}=9.6 \times 10^{32} \mathrm{~cm}^{-2} \mathrm{~s}^{-1}, \sqrt{s}=7 \mathrm{TeV}$.

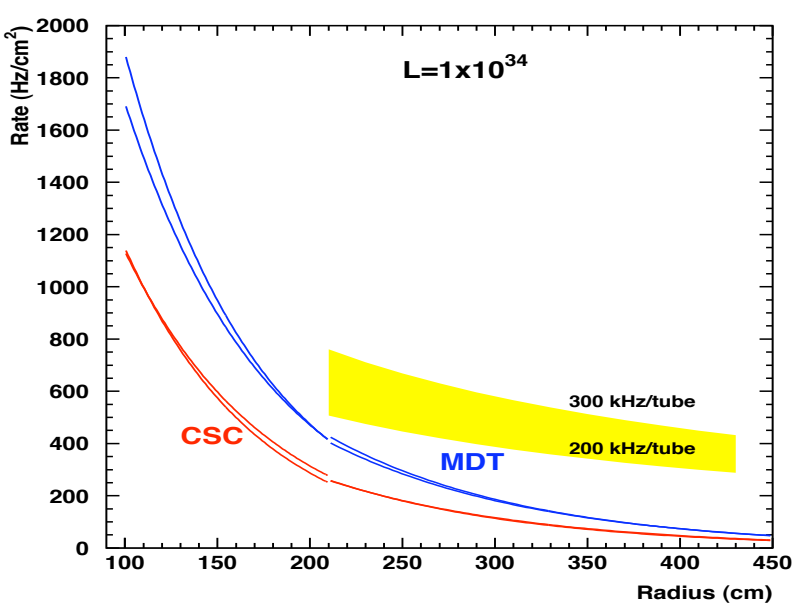

(b) Scaled to $\mathcal{L}=1 \times 10^{34} \mathrm{~cm}^{-2} \mathrm{~s}^{-1}, \sqrt{s}=7 \mathrm{TeV}$.

Fig. 4. Measured Cavern background at the level of the muon Small Wheel. Figure (a) shows measurements and a simulation estimation of the background. Given the small discrepancy between measurements and simulation the measured values have been used for subsequent extrapolation to higher luminosities. Figure (b) shows an extrapolation to a luminosity of $\mathcal{L}=1 \times 10^{34} \mathrm{~cm}^{-2} \mathrm{~s}^{-1}, \sqrt{s}=7 \mathrm{TeV}$. [4]

The expected hit rate at the maximum Phase-II luminosity of $7 \times 10^{34} \mathrm{~cm}^{-2} \mathrm{~s}^{-1}$ will be $\sim 14 \mathrm{kHz} / \mathrm{cm}^{2}$ assuming conservatively the MDT sensitivity for the future detector. The extrapolation to high luminosities is simple as the rate of cavern background is proportional to the beam luminosity. This has been established experimentally by studying hit rates and currents in several muon detectors at LHC runs of various luminosities. All the above is based on the measurements at 
$\sqrt{s}=7 \mathrm{TeV}$. The ratio of background rate at $\sqrt{s}=14 \mathrm{TeV}$ and $7 \mathrm{TeV}$ is about 1.3 in the Small Wheel region according to the Monte Carlo simulation using FLUGG.

Any new detector that might be installed in the place of the current Small Wheel should be operational for the full life time of ATLAS (and be able to integrate $3000 \mathrm{fb}^{-1}$ ). Assuming 10 years of operation and the above expected hit rate per second, approximately $10^{12}$ hits $/ \mathrm{cm}^{2}$ are expected in total in the hottest region of the detector [6].

The proposed detectors should be validated for ageing effects at a level of about 1 Coulomb $/ \mathrm{cm}^{2}$ (or higher) for a planar detector or the equivalent for a wire chamber, and in case the required performance is difficult to achieve, the possibility of replacing chambers, especially in the very forward region, should be envisaged. The trigger and read-out electronics should be also be validated in the same conditions.

\section{B. Physics performance and precision tracking}

It has been shown that the current muon detectors in the NSW region cannot operate efficiently in the high background environment expected with the various LHC upgrades. A new detector needs to be built which will give high detection and reconstruction efficiency, even at the highest particle fluxes expected. The performance of the new detector at high luminosity should be at least as good as that of the current detector at low luminosity, and be able to measure the transverse momentum $\left(p_{T}\right)$ of passing muons with a precision of $10 \%$ for $1 \mathrm{TeV}$ muons in the full pseudorapidity ${ }^{1}$ coverage of the Small Wheel (up to $\eta=2.7$ ). In particular such a detector should have the following characteristics:

- Measure hits with a position resolution in the bending plane of $<100 \mu$ m per measurement;

- Angular resolution of $\sim 0.3 \mathrm{mrad}$;

- Segment finding efficiency of more than $97 \%$ for muons with $p_{T}$ more than $10 \mathrm{GeV}$ (the segment finding efficiency for the current MDT system), and track segment resolution of less than $50 \mu$ m including all alignment and calibration errors;

- Efficiency and resolutions should not degrade at very high momenta (due to $\delta$ rays, showers etc);

- Measure the second coordinate with a resolution of 1 $2 \mathrm{~mm}$.

\section{Trigger selection power}

Trigger simulations show that selecting muons with $p_{T}>$ 20 at Level-1 (L1MU20) one would get a trigger rate at $\sqrt{s}=14 \mathrm{TeV}$ and at an instantaneous luminosity of $3 \times$ $10^{34} \mathrm{~cm}^{-2} \mathrm{~s}^{-1}$ of approximately $60 \mathrm{kHz}$, to be compared to the total allowed Level-1 rate of $100 \mathrm{kHz}$. Due to the limited $p_{T}$ resolution of the Level-1 muon trigger system, raising the

\footnotetext{
${ }^{1}$ ATLAS uses a right-handed coordinate system with its origin at the nominal interaction point (IP) in the centre of the detector, and the z-axis along the beam line. The $\mathrm{x}$-axis points from the IP to the centre of the LHC ring, and the $\mathrm{y}$-axis points upwards. Cylindrical coordinates $(r, \phi)$ are used in the transverse plane, $\phi$ being the azimuthal angle around the beam line. Observables labelled transverse are projected into the xy plane. The pseudorapidity $\eta$ is defined in terms of the polar angle $\theta$ as $\eta=-\ln [\tan (\theta / 2)]$.
}

threshold above $20 \mathrm{GeV}$ does not further improve significantly the signal to noise ratio.

In order to reduce the low $p_{T}$ components, the $p_{T}$ resolution of the muon Level-1 system needs to be heavily improved. A precision angle measurement at the Small Wheel level can be used to improved the $p_{T}$ resolution of the Level-1 trigger in the end-cap. In the present layout, the contributing factors to the $p_{T}$ resolution are: i) angular resolution of the current Big Wheel trigger station $(\sim 3 \mathrm{mrad})$, ii) multiple scattering in the end-cap toroid ( $\sim 0.5 \mathrm{mrad}$ for high $p_{T}$ muons), iii) multiple scattering in the calorimeters ( $\sim 2$ to $3 \mathrm{mrad}$ for low energy muons and considerably less than $1 \mathrm{mrad}$ for high energy ones), and iv) finite size of luminous region of $p$ - $p$ collision (corresponds to 1-2 mrad depending on $\eta$ ). A precision angle measurement by the Small Wheel can be used to eliminate the contributions iii) and iv) by correcting for these effects track by track, thus removing a part of the smearing effects to improve the $p_{T}$ resolution. To be compatible with the performance of the Big Wheel, a resolution of $3 \mathrm{mrad}$ or better is required for the determination of the track segment angle (angle relative to the pointing direction to the IP) at the level of the Small Wheel. However the ATLAS Phase-II upgrade will probably improve part of the Big Wheel trigger resolution to $1 \mathrm{mrad}$. Hence a similar resolution of $1 \mathrm{mrad}$ is required at the level of the Small Wheel for a detector that will have to perform adequately for the whole lifetime of the ATLAS experiment.

\section{System OVERVIEW}

The NSW system consists of sTGC and micro megas (MM) detectors. Figure 2 shows the location of the NSW with respect to the Big Wheel, and the end-cap toroid magnets. Each technology is composed of eight detection layers. There are two sTGC quadruplets in the outer layers and inside are two MM layers. Although both technologies can provide both trigger and precision measurements, the sTGC is the primary trigger and the MM are mainly used for precision measurement. The NSW trigger system provides candidate muon tracks to the TGC Sector Logic which uses them to corroborate trigger candidates from the Big Wheel TGC chambers. The Sector Logic then sends Level-1 trigger candidates to the ATLAS Muon Central Trigger logic. The radial coordinate of tracks found in the NSW is measured by high precision strips. The azimuthal, $\phi$ coordinate is determined by the triggering tower of NSW pads for sTGC and by small angle stereo strips for the MM. A line from the interaction point through the $R-\phi$ point of a track in the NSW is projected onto the Big Wheel's $R-\phi$ array of Regions-of-Interest (RoI). Tracks within a $\pm 7.5 \mathrm{mrad}$ angle of this line are passed to the Sector Logic which attempts to match it to an active RoI in the Big Wheel. Confirmed hits become Level-1 candidates sent to the Muon Central Trigger Interface.

In addition to a precision track coordinate for projection to the Big Wheel, the NSW trigger measures the polar angle of the track inside the Small Wheel, i.e. before the end-cap toroid, to an accuracy of $<1 \mathrm{mrad}$. This is done by calculating the track position in two virtual planes within the Small Wheel. For the sTGC these are located on either side of the MM 
chambers separated by about $30 \mathrm{~cm}$ (See Fig. 2.). For MM the separation is less, resulting in poorer pointing resolution. For sTGC, each of these two radial coordinates is calculated by averaging up to four centroids of strip signals from four sTGC detectors. The centroids are calculated by the sum of strip charges weighted by position. Finally, the radial position and angle $\theta$, between a track in the NSW and an infinite momentum track from the interaction point, are calculated from these two coordinates. The azimuthal coordinate, $\phi$, is defined by the pad tower that triggered the sTGC trigger. For the MM we take advantage of the $0.5 \mathrm{~mm}$ strip pitch and use the strip address of the earliest arriving hit in a given bunch crossing to obtain the radial coordinate with sufficient resolution in each plane in order to calculate the polar angle. The $\phi$ coordinate is obtained by a lookup table addressed by the strip addresses of two small angle stereo views. A stereo angle of just $3^{\circ}$ is sufficient to determine the $\phi$ coordinate with the required precision. The angle $\theta$ is passed to the Sector Logic, but will not be used until Phase-II when the New Big Wheel trigger will provide an angle of similar accuracy; the two angles can then be used to calculate a much more accurate $p_{\mathrm{T}}$ for the Level-1 trigger than is currently. For Phase-I the improved $p_{\mathrm{T}}$ can be calculated by Level-2 using MDT data.

The sTGC NSW trigger logic uses pad tower triggers to drastically reduce the amount of sTGC data to be processed on each bunch crossing for the Level-1 trigger. Three-out-of-four coincidences are made in pad towers in each sTGC quadruplet; these are then combined to choose a band of strips in each of the eight sTGC layers to be readout to the trigger processors in USA $15^{2}$. The details are described in Section VII.

There are no plans to use the wires in the sTGC trigger, but they are read out in response to a Level-1 Accept and are therefore available to the Level-2 trigger. Only the outer two detectors of each layer have wire readout. For the inner two, the pad segmentation provides a fine enough $\phi$ coordinate.

\section{STGC DETECTOR TECHNOLOGY}

The basic Small strip Thin Gap Chamber (sTGC) structure is shown in Fig. 5[a]. It consists of a grid of $50 \mu \mathrm{m}$ gold plated tungsten wires with a $1.8 \mathrm{~mm}$ pitch, sandwiched between two cathode planes at a distance of $1.4 \mathrm{~mm}$ from the wire plane. The cathode planes are made of a graphite-epoxy mixture with a typical surface resistivity of $100 \mathrm{k} \Omega / \square$ sprayed on a $100 \mu \mathrm{m}$ thick G-10 plane, behind which there are on one side strips (that run perpendicular to the wires) and on the other pads (covering large rectangular surfaces), on a $1.6 \mathrm{~mm}$ thick PCB with the shielding ground on the opposite side (see Fig. 5[b]). The strips have a $3.2 \mathrm{~mm}$ pitch, much smaller than the strip pitch of the ATLAS TGC, hence the name 'Small TGC' for $t$ his technology.

A similar type of structure was used in the past for the OPAL Pole-Tip calorimeter, where 400 detectors were constructed and run for 12 years.

\footnotetext{
${ }^{2}$ The USA15 cavern next to the ATLAS experimental cavern hosts most of the electronics in the experiment and it is accessible during the runs of the LHC.
}

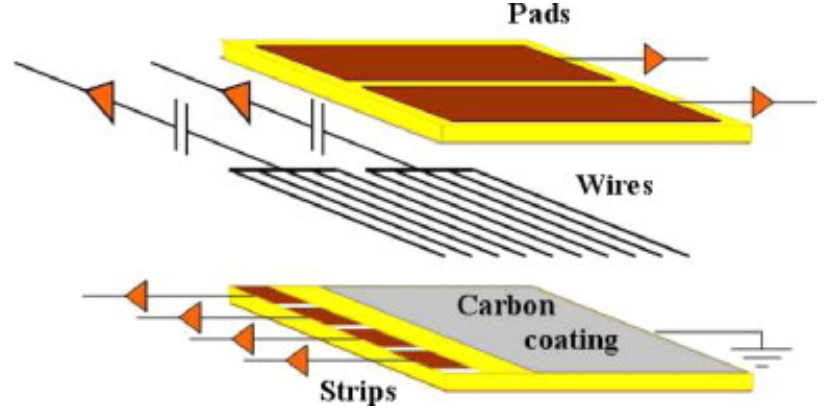

(a) a

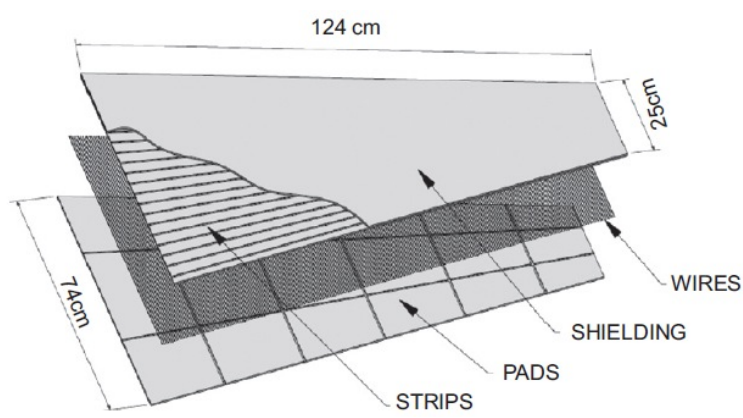

(b) $\mathrm{b}$

Fig. 5. The sTGC internal structure.

\section{STGC TRIGGER SYSTEM}

At each bunch crossing, the sTGC trigger electronics finds local tracks that point, with $<1 \mathrm{mrad}$ precision, to the Big Wheel to corroborate its coincidences. In order to place the track finding and extrapolation logic off-detector, we use a 3out-of-4 coincidence of pads in each of the 4-layer quadruplets to choose the relevant bands of strips to be sent off-detector. This reduces both the required bandwidth and the amount of centroid and track finding logic. To reduce the number of pad channels, the layers are staggered by half a pad in both directions to make logical towers one quarter the area of a pad.

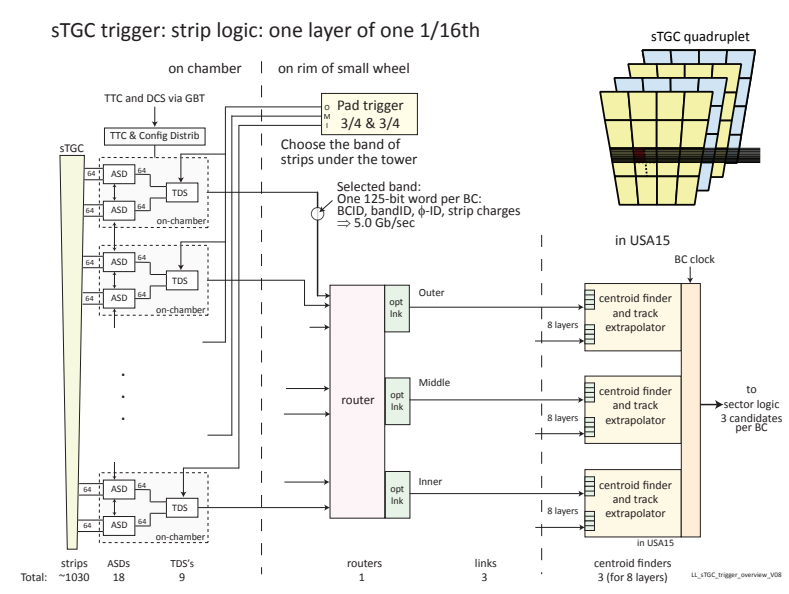

Fig. 6. Block diagram of the trigger logic for one layer (of eight layers per side) of a $1 / 16^{t h}$ sector

The block diagram of the trigger logic for one layer of a 
$1 / 16^{t h}$ sector is shown in Fig. 6 . The figure shows the signal flow from chamber to the track finding and extrapolation logic in USA15. On detecting a peak in the signal from a strip, the $\mathrm{VMM}^{3}$ digitizes the peak value to six bits which are sent serially on a line dedicated to each channel to the Trigger Data Serializer (TDS) companion ASIC. On each beam crossing clock, the charges of all strips are captured with a fixed latency from the beam crossing. Meanwhile the pad coincidence logic selects the bands of strips in each layer that pass through the tower generating the coincidence. Currently, up to three such bands per layer, one per Inner/Middle/Outer region, can be selected per beam crossing. The number of regions and their boundaries that balance the probability of trigger candidates will be optimized with simulations.

The IDs of the bands that are selected are distributed to the TDS chips. The one in each layer of each region that holds a selected band transmits the strip charges on a fast serial copper pair to a router on the periphery of the wheel. The data transmitted include the Bunch Crossing ID, band-ID, $\phi$-ID and the strip charges. 128 bits must be transmitted in $25 \mathrm{nsec}$, necessitating a link with $5 \mathrm{~Gb} / \mathrm{sec}$ payload. The Router then sends the data from the active TDS ASICs to the centroid finding and track extrapolation logic (see section VII-3) in USA15 via an optical fibre.

In USA15 the active strips selected by the pad trigger are used to compute centroids and then track segments that point to the Big Wheel. These candidates are then sent via fast serial links to the Sector Logic to be combined with Big Wheel candidates. The whole process repeats every bunch crossing with a fixed latency (See Table II). Details are elaborated in the following sections.

1) Pad trigger and random trigger rates: The pad tower trigger tags a bunch crossing and the band of strips passing through the triggered tower in each layer that must be selected for readout by the TDS ASIC. Its inputs are all the $\sim 1300$ $(\sim 1200)$ pads of all 8 layers in a large (small) $1 / 16^{t h}$ sector, up to $|\eta|<2.4$. It is located on the periphery of the Small Wheel and must be implemented in radiation tolerant electronics. The $1 / 16^{\text {th }}$ sector is divided into regions in radius (currently three, without regard to physical gas volumes) of roughly balanced trigger rate. Pad tower triggers are produced independently in each region so that one candidate from each region can be sent to the Big Wheel Sector Logic. The BCID and $\phi$ of the triggering pad tower are also sent to the selected TDS and flow downstream along with the selected digitized strip charges. It is the pad trigger that defines to which bunch crossing the strip signals belong. The logic is as follows:

- make a 3-out-of-4 coincidence for every tower in each quadruplet.

- make a coincidence between the 3-out-of-4 coincidences in the two quadruplets.

- choose up to one coincidence per Inner/Middle / Outer region.

\footnotetext{
${ }^{3}$ The VMM is a configurable, radiation hard front-end ASIC, developed for both the MM and sTGC detectors, that incorporates the analog circuitry for reading out particle detectors, time and amplitude digitizers and digital logic for buffering the data and transmitting it out on-demand.
}

TABLE II

LATENCY FROM THE INTERACTION POINT, THROUGH THE STGC TRIGGER LOGIC FOR THE NSW TO THE SECTOR LOGIC IN USA-15. ALL TIMES ARE ESTIMATES, EXCEPT FOR THE CENTROID FINDER AND THE VMM1 LATENCY WHICH HAVE BEEN MEASURED.

\begin{tabular}{|c|c|c|c|}
\hline & $\begin{array}{l}\min \\
(\mathrm{ns})\end{array}$ & $\begin{array}{r}\max \\
(\mathrm{ns})\end{array}$ & Notes \\
\hline $\begin{array}{l}\text { TOF from interaction point } \\
\text { to NSW }(\mathrm{z}=7.8 \mathrm{~m})\end{array}$ & 29 & 31 & to periphery of NSW @ R=5m \\
\hline pad signal jitter in chamber & 25 & 25 & $\begin{array}{l}\text { worst case due to tracks } \\
\text { midway between wires }\end{array}$ \\
\hline pad ASD (VMM) & 55 & 65 & VMM1 latency + time-to-peak \\
\hline serialize 32 pads @2 GHz & 16 & 20 & \\
\hline TDS to pad trigger on rim, $\max 4 \mathrm{~m}$ & 15 & 20 & $3-4 \mathrm{~m} @ 5 \mathrm{~ns} / \mathrm{m}$ \\
\hline up- to down-stream package $(50 \mathrm{~cm})$ & 3 & 3 & \\
\hline pad trigger (incl de-skew) & 15 & 25 & strip charge values are pipelined \\
\hline pad trigger to on-chamber TDS ASIC & 15 & 20 & 3-4m@5nsec/m \\
\hline TDS: Trigger Data Serializer & 10 & 20 & until pad trigger arrives \\
\hline On chamber cabling to Router & 15 & 20 & $5 \mathrm{nsec} / \mathrm{m}, 3$ to $4 \mathrm{~m}$ \\
\hline TDS-to-Router ser/deserializer & 15 & 25 & \\
\hline Router & 6 & 10 & switch \\
\hline Router to Centroid deserializer & 40 & 70 & $\begin{array}{l}\text { GBT does not meet this limit. } \\
\text { A different link must be used. }\end{array}$ \\
\hline fibre to Centroid card in USA $1590 \mathrm{~m}$ & 450 & 450 & $5 \mathrm{nsec} / \mathrm{m}$ \\
\hline find valid centroids and averages & 32.5 & 52 & $\begin{array}{l}8 \text { layers done in parallel, } \\
\text { measured to be } 13 \text { clocks }\end{array}$ \\
\hline difference of centroid aver & 2.5 & 4 & \\
\hline lookup sub-ROI pointed to at $\mathrm{BW}$ & 5 & 5 & \\
\hline $\begin{array}{l}\text { resynch to BC clock driving the } \\
\text { output serializer }\end{array}$ & 6.25 & 6.25 & $\begin{array}{l}90^{\circ} \text { phase chosen to best match } \\
\text { pipeline length }\end{array}$ \\
\hline centroid to Sector Logic serializer & 5 & 15 & $\begin{array}{l}\text { deserializer on Sector } \\
\text { Logic latency budget }\end{array}$ \\
\hline Total & 760 & 886 & \\
\hline
\end{tabular}

The Trigger Data Serializer chip will latch the pad signal from 32 pads on every beam crossing. These 32 bits are then serialized at $2 \mathrm{~Gb} / \mathrm{s}$ (i.e. 32 bits can be transmitted as $8 \mathrm{~b} / 10 \mathrm{~b}$ in less than $25 \mathrm{~ns}$ ) and sent on a single pair to the Pad Logic. This reduces the number of cables to approximately 64 per sector. Although this is a fair number of cables, it preserves the flexibility of doing the pad tower coincidence logic in accessible programmable logic.

2) Simple pad trigger simulation: In order to verify the sTGC trigger algorithm and to evaluate its efficiencies and fake rates at various levels, a fast simulation program has been developed. Its main features are:

- Detector and pad geometry: the exact geometry taken from the parameter book. In particular the pads staggering and displacement is faithfully reproduced.

- Magnetic field and multiple scattering: an angle distributed with a Gaussian distribution with $\sigma_{\eta}, \sigma_{\phi}=5,20 \mathrm{mrad}$ is conservatively assumed.

- Incoherent Background: the $\eta$-dependent incoherent rate is from Fig. 4, scaled to $\mathcal{L}=5 \times 10^{34} \mathrm{~cm}^{-2} s^{-1}$.

- Coherent Background: the 'standard' coherent background remains to be simulated.

- Cathode charge distribution: a Gaussian distribution with $\sigma=3 \mathrm{~mm}$ is assumed. The charge is spread in two dimensions over the neighbouring pads.

- Efficiency: an efficiency of 1 for a charge deposit above $0.5 \mathrm{mip}$ is assumed. For a deposit below $0.2 \mathrm{mip}$ the efficiency is null, and increases linearly between these two values.

It should be noted that each of the above features with its parameters can be switched on or off and parameterized. Once energy deposition and digitization have been performed, all 3 -out-of-4 hit coincidences in pad towers in each wedge and the combined coincidence of the two 3-out-of-4 hit wedge 
coincidences are found. Table III summarizes the results. Note that the sector trigger rate depends on the $6^{\text {th }}$ power of the individual rate.

TABLE III

RESULTS OF THE SIMPLE PAD TRIGGER SIMULATION

\begin{tabular}{|c|c|c|c|c|c|c|c|c|c|c|}
\hline & \multicolumn{3}{|c|}{ Small Sector } & \multicolumn{3}{|c|}{ Large Sector } & \multicolumn{4}{|c|}{ Total } \\
\hline & Pivot & onfirm & Total & Pivot & nfirm & Total & & octant & side & 2 sides \\
\hline Hits per $B C$ & 7.0 & 7.4 & 14.5 & 9.4 & 9.8 & 19.3 & & 33.7 & 269.7 & 539.3 \\
\hline $\begin{array}{l}\text { Hits per BC for } \\
|\eta|<2.4\end{array}$ & 6.0 & 5.9 & 11.9 & 7.9 & 8.5 & 16.4 & & 28.3 & 226.4 & 452.7 \\
\hline $\begin{array}{l}\text { Pad occupancy } \\
\text { per BC }\end{array}$ & $1.0 \%$ & $1.0 \%$ & $1.0 \%$ & $1.1 \%$ & $1.3 \%$ & $1.2 \%$ & & $1.1 \%$ & $1.1 \%$ & $1.1 \%$ \\
\hline $\begin{array}{l}\text { 3-out-of-4 wedge } \\
\text { rate, } \mathrm{kHz}\end{array}$ & 2043 & 2213 & 4256 & 3008 & 3166 & 6175 & $\mathrm{MHz}$ & 10.4 & 83.4 & 166.9 \\
\hline $\begin{array}{l}\text { Sector trigger } \\
\text { rate, } \mathrm{Hz}\end{array}$ & & & 364.0 & & & 645.4 & $\mathrm{kHz}$ & 1.0 & 8.1 & 16.1 \\
\hline
\end{tabular}

3) Centroid and track finder: Centroid finding, averaging and extrapolation to the Big Wheel will be done in a conventional FPGA in USA15. The centroid finder demonstrator calculates the centroid from the charge on up to five strips. Centroids are included in the average only if the number of strips above threshold is below a certain maximum value (e.g. five) in order to exclude $\delta$-rays and conversions. Fig. 7 shows the details of the centroid finding and averaging calculation.

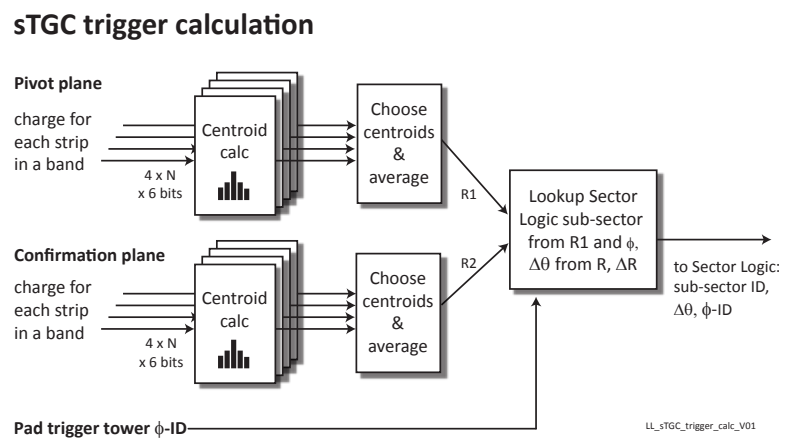

(a) Block diagram of the centroid calculation, centroid averaging and extrapolation to the Region-of-Interest, RoI, in the Big Wheel.

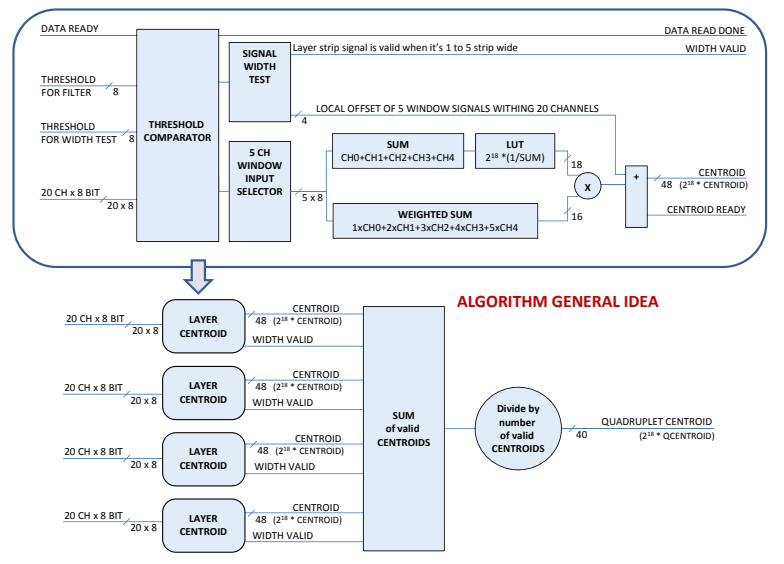

(b) Details of the centroid algorithm

Fig. 7. The centroid algorithm

The identification of the track in the NSW with a RoI at the Big Wheel is done using Look-up-Tables (LUTs) for the radius, $\mathrm{R}$, and azimuth, $\phi$, coordinates. The LUT input for $\mathrm{R}$ is the centroid average in the pivot plane; the LUT input for $\phi$ is the column of the triggering pad tower. The algorithm also provides the Sector Logic with the angle, $\theta$, between the track in the NSW and an infinite momentum track from the interaction point, calculated from the two centroid averages in the two quadruplets.

\section{READ-OUT SYSTEM AND DATA-QUALITY MONITORING}

The off-detector readout chain of the ATLAS detectors presently consists of sub-detector specific ReadOut Drivers(RODs), which output event fragments to the ReadOut System (ROS) via the ReadOut Links (ROLs) [1]. The ROLs are point-to-point links in the form of optical fibres; the protocol used is the S-link protocol [7]. The ROLs cross the boundary between the sub-detector domains and the TDAQ domain. After Long Shutdown 3 this organisation will be changed: the working group for defining the architecture of the off-detector readout has recommended to split the ROD functionality in two:

- The front-end links connecting the detectors to the offchamber readout electronics will be interfaced to a general purpose COTS network with a stateless, non-detectorspecific and high-availability interface.

- The ROD data processing and other functions will operate on data delivered by an industry standard network. Eventually such a ROD could be implemented in an industry standard PC.

The front-end links will be for many subdetectors by CERN GBT links [8], which also can, and will, be used for transferring TTC, DCS, and front-end configuration information. In view of the handling of DCS traffic, the interface should be as stateless and as ignorant of detector specifics as possible, that is, its state should not depend on the state of the DAQ system and subdetector-specific data handling should be avoided. The interface must also be able to generate a BUSY signal when its event data buffers become full and to connect to both the current and the upgraded Phase-II TTC system.

The connectivity of the interface, which is named FELIX (Front End LInk eXchange) will be implemented with FPGAs for interfacing to the GBT links and for formation of data streams that will be routed to server PCs. For event data, these PCs will perform the classic ROD functions: fragment building and associated error detection, data checking and transformation and monitoring. The fragments may be forwarded to server PCs implementing the ROS functionality or alternatively the ROS functionality can also be implemented in the same server PCs as used for fragment building. DCS and front-end configuration interact with the on-chamber systems via the general purpose network and FELIX.

Two important advantages of the architecture are: minimization of the amount of special purpose detector-specific hardware and associated firmware and the flexibility offered by the use of general purpose, industry standard networking and server PCs. On the time scale of installation 40 or $100 \mathrm{~Gb} / \mathrm{s}$ Ethernet (and similar speeds for Infiniband) can be expected to be available at reasonable cost, so that the data from 10 to 25 fully utilized GBT links can be concentrated on a single network link (even 
higher concentration is possible when, as for the NSW, the GBT bandwidth is not be fully utilized).

These data can then be distributed over as many server PCs taking care of the traditional ROD functionality as needed. If needed, the processing capabilities of these PCs could be augmented by GPUs or other types of coprocessors such as the Intel Xeon Phi coprocessor [9]. The use of PC technology and COTS network (for the output to the ROS as well) will make it possible to purchase the hardware relatively late, benefiting from further evolution of the technology. Testbeam work and production testing will be possible with less performant network and server PC hardware than needed for the final system, but the network protocols and the software can be identical to those to be used in the final system.

By adopting FELIX as the NSW off-detector readout, the NSW will be Phase-II compatible with respect to functionality ${ }^{4}$. There are two possible models for FELIX: (1) a server PC with PCI-express plug-in cards: one or more FPGAs cards interfacing to GBT links and one network card, or (2) a completely FPGA implementation. In both, the interface to the TTC system is by means of mezzanines that can be replaced for the Phase-II TTC system. Although the former is less compact, its network connection does not require firmware development, it is more flexible and is more easily upgradeable. The FPGA boards maybe commercial boards or boards developed for other projects such as the ATCA based TELL40 for the LHCb Phase 1 DAQ upgrade [10], the ALICE C-RORC PCIe board [11], to be used as the new ROBIN [12] for Phase 0 ATLAS DAQ and the GLIB board [13] developed by the CERN GBT project. For initial testing of the FELIX approach in 2013/2014, the server PC-plus-PCIe plugin card model will be taken. Either the C-RORC, the GLIB board if its PCIe interface can be used, or a commercial FPGA PCIe card will be used. NSW collaborators will be part of a wider collaboration designing and building the FELIX interface.

\section{CONCLUSION}

The proposed NSW will allow the ATLAS muon system to maintain its full trigger acceptance and its excellent muon tracking at the highest LHC luminosities expected. At the same time the Level-1 single muon (typically $p_{T}>20 \mathrm{GeV}$ ) triggers rate will be kept at an acceptable level. The design of the NSW meets the requirement for very good segment angle resolution of $1 \mathrm{mrad}$ at the Level-1 trigger level. This angular resolution allows for a powerful background rejection in the track density NSW environment. It is also an important step towards a further improvement of the muon Level-1 trigger system foreseen in the Phase-II upgrade for even higher luminosity. This PhaseII upgrade will substantially improve the $p_{T}$ resolution of the Level-1 muon system. This will be achieved by using the information of precision tracking detector (Monitored Drift Tubes, MDT) as part of the EM trigger system and by combining with that the precision angle per track segment provided by the NSW.

\footnotetext{
${ }^{4}$ Full Phase-II compatibility requires the hardware to be performant enough and to support interfacing to a new TTC system.
}

The NSW project consists of R\&D of detector technology and electronics. The schedule for installation is 2018 at LS2. Before the installation, it is planned that the completed wheels are fully tested on surface during 2017 for about one year. In order to match such a schedule, the production of the chamber multiplets, which is expected to take $<2$ years to complete, should start from 2015. Year 2014 will be devoted to the production of module- 0 and its qualification.

\section{REFERENCES}

[1] ATLAS Collaboration, The ATLAS Experiment at the CERN Large Hadron Collider, JINST 3 (2008) S08003.

[2] ATLAS Collaboration, Letter of Intent for the Phase-I Upgrade of the ATLAS Experiment, Tech. Rep. CERN-LHCC-2011-012. LHCC-I-020, Geneva, Nov, 2011.

[3] ATLAS Muon Spectrometer: Technical Design Report. Technical Design Report ATLAS. CERN, Geneva, 1997. CERN/LHCC/97-22.

[4] L. Jeanty, "Data and mc comparisons for muon spectrometer, including cavern background." Presentation at the lpcc simulation workshop, October, 2011. http://indico.cern.ch/getFile.py/access?contribId= $3 \&$ sessionId=0\&resId=0\&materialId=slides \& confId $=144956$.

[5] P. Sala and A. Vannini, "The flugg project." http://www.fluka.org/content/tools/flugg/, 2013.

[6] ATLAS Collaboration, Letter of Intent for the Phase-II Upgrade of the ATLAS Experiment, Tech. Rep. CERN-LHCC-2012-022. LHCC-I-023, Geneva, Dec, 2012. Draft version for comments.

[7] H. Van der Bij, R. McLaren, O. Boyle, and G. Rubin, S-LINK, a data link interface specification for the LHC era, IEEE Trans. Nucl. Sci. 44 no. 3, (1997) 398-402.

[8] T. G. Team, “The gbt project.” https://cds.cern.ch/record/1235836?ln=en, 2013.

[9] "Intel xeon phi coprocessor." http://software.intel.com/en-us/mic-developer.

[10] H. Dijkstra and U. Kebschull, The LHCb Upgrade, ECONFC070512:027,2007 (2007)

[11] H. Engel and Kothers, ALICE CRORC as CBM FLES Interface Board Prototype, GSI SCIENTIFIC REPORT 2011 (2011).

[12] R. Cranfield et al., The ATLAS ROBIN, JINST 3 T01002 (2008).

[13] P. Vichoudis et al., The Gigabit Link Interface Board (GLIB), a flexible system for the evaluation and use of GBT-based The Gigabit Link Interface Board (GLIB), a flexible system for the evaluation and use of GBT-based optical links, JINST 5 (2010) C11007.

Yonathan Munwes received is B.S.c from Tel Aviv University in physics and geophysics in 2007. Yonathan continued his studies toward PhD in experimental HEP at Tel Aviv University. During his $\mathrm{PhD}$ he was part of the israeli group that developed the new sTGC for the NSW project. In addition he is carrying physics analysis within the ATLAS experiment at the LHC. 